\title{
An Induction-Based Magnetohydrodynamic 3D Code for Finite Magnetic Reynolds Number Liquid-Metal Flows in Fusion Blankets
}

\author{
Charlie Kawczynski ${ }^{\mathrm{a}}$, Sergey Smolentsev ${ }^{\mathrm{a}, *}$, Mohamed Abdou $^{\mathrm{a}}$ \\ ${ }^{a}$ UCLA, MAE Department, 44-114 Engineering IV, 420 Westwood Plaza, Los Angeles, CA 90095-1597, USA
}

\begin{abstract}
Most numerical analysis performed in the past for MHD flows in liquid-metal blankets were based on the assumption of low magnetic Reynolds number and involved numerical codes that utilized electric potential as the main electromagnetic variable. One limitation of this approach is that such codes cannot be applied to truly unsteady processes, for example, MHD flows of liquid-metal breeder/coolant during unsteady events in plasma, such as major plasma disruptions, edge-localized modes and vertical displacements, when changes in plasmas occur at millisecond timescales. Our newly developed code MOONS (Magnetohydrodynamic Object-Oriented Numerical Simulation) uses the magnetic field as the main electromagnetic variable to relax the limitations of the low magnetic Reynolds number approximation for more realistic fusion reactor environments. The new code, written in Fortran, implements a 3D finite-difference method and is capable of simulating multi-material domains. The constrained transport method was implemented to evolve the magnetic field in time and assure that the magnetic field remains solenoidal within machine accuracy at every time step. Various verification tests have been performed including purely hydrodynamic flows and MHD flows at low and finite magnetic Reynolds numbers. Test results have demonstrated very good accuracy against known analytic solutions and other numerical data.
\end{abstract}

Keywords: liquid-metal blanket, magnetohydrodynamics, numerical code, induced magnetic field, magnetic Reynolds number

\section{Introduction}

Magnetohydrodynamic (MHD) codes are important tools for design and analysis of liquid-metal blankets [1]. Induced magnetic fields are often dismissed as negligible compared to external magnetic fields in many codes used to simulate liquid-metal MHD flows in fusion reactor environments, but this is not always the case. Major plasma disruptions, edge-localized modes and vertical displacements may result in strong electromagnetic interactions in the liquid-metal, which cannot be described with the inductionless approximation. In addition, magnetic Reynolds number $\left(R e_{m}\right)$ based on large duct lengths (e.g. long poloidal "banana" segments) may yield moderate values comparable with unity.

\footnotetext{
* Corresponding author

Email address: sergey@fusion.ucla.edu (Sergey Smolentsev )

Preprint submitted to Fusion Engineering and Design
}

The magnetic induction (B) formulation, based on utilization of the magnetic field as the main electromagnetic variable, is more general and has several advantages over the electric potential $(\varphi)$ based formulation. Induced magnetic fields and its transport are captured in the B-formulation but ignored in the $\varphi$-formulation. Another advantage of the Bformulation is that conservation of charge, a challenging and important constraint in MHD computations [2], is automatically enforced if the magnetic field is numerically solenoidal. The goal of this paper is to introduce a new induction-based code for finite $R e_{m}$ MHD flows and present test results and its capabilities.

\section{Mathematical Formulation}

Combining Ohm's law, Faraday's law, and the Ampère-Maxwell equation yields the induction equation. Momentum, continuity and induction

February 1, 2016 
equations are non-dimensionalized by characteristic velocity, $U$, length, $L$, time, $L / U$, pressure, $\rho U^{2}$, and magnetic field, $B$. Assuming incompressible and isothermal conditions, the dimensionless momentum, induction, mass continuity and magnetic field continuity equations are:

$$
\begin{gathered}
\frac{\partial \mathbf{u}}{\partial t}+\nabla \cdot(\mathbf{u u})=-\nabla p+\frac{1}{R e} \nabla^{2} \mathbf{u} \\
+\frac{H a^{2}}{R e R e_{m}}\left(\nabla \times \mathbf{B}^{i n d}\right) \times\left(\mathbf{B}^{0}+\mathbf{B}^{i n d}\right), \\
\frac{\partial\left(\mathbf{B}^{0}+\mathbf{B}^{i n d}\right)}{\partial t}-\nabla \times\left\{\mathbf{u} \times\left(\mathbf{B}^{0}+\mathbf{B}^{i n d}\right)\right\}+ \\
\frac{1}{R e_{m}} \nabla \times\left\{\frac{1}{\bar{\sigma}} \nabla \times\left(\frac{\mathbf{B}^{0}+\mathbf{B}^{i n d}}{\bar{\mu}_{m}}\right)\right\}=0, \\
\nabla \cdot \mathbf{u}=0, \quad \nabla \cdot \mathbf{B}=0 .
\end{gathered}
$$

Here, $\mathbf{u}, p, \mathbf{B}$ are dimensionless velocity, pressure and magnetic field (separated into induced, $\mathbf{B}^{\text {ind }}$, and applied, $\mathbf{B}^{0}$ ) respectively. In Eqs. (1) and (2), Reynolds number ( $R e$ ) Hartmann ( $H a$ ) magnetic Reynolds number $\left(R e_{m}\right)$ and dimensionless ratios of electrical conductivity and magnetic permeability are defined as

$$
\begin{gathered}
R e=\frac{U L}{\nu}, \quad H a=B L \sqrt{\frac{\sigma}{\rho \nu}}, \quad R e_{m}=\mu_{m} \sigma U L, \\
\bar{\sigma}=\left\{\begin{array}{ll}
1 & \text { fluid } \\
\frac{\sigma_{w}}{\sigma} & \text { wall }
\end{array}, \quad \bar{\mu}_{m}= \begin{cases}1 & \text { fluid } \\
\frac{\mu_{m, w}}{\mu_{m}} & \text { wall }\end{cases} \right.
\end{gathered}
$$

Here, $\nu, \rho, \sigma, \mu_{m}$ are kinematic viscosity, density, electrical conductivity and magnetic permeability of the fluid while $\sigma_{w}$ and $\mu_{m, w}$ pertain to the wall respectively. Reynolds number is the ratio of inertial to viscous forces. Hartmann number squared is the ratio of electromagnetic to viscous forces. Magnetic Reynolds number is the ratio of magnetic field convection to diffusion. In this study $\bar{\mu}_{m}$ is assumed to be unity.

Similar to Eq. (3), Eq. (4) is a physical constraint on the magnetic field to remain solenoidal. Mathematically, Eq. (4) does not need to be solved along with Eq. (2) but this may lead to unphysical forces in the momentum equation $[3,4]$. The constrained transport (CT) method is used to enforce Eq. (4) and will be discussed in Section 3.
All solved variables begin with zero magnitude. Typical boundary conditions (BC) for velocity and pressure are Dirichlet, Neumann and periodic. The most physically reliable BCs for the magnetic field is $\mathbf{B}=\mathbf{0}$ far from the flow domain. In practice, this can require many grid points and be computationally expensive. Several methods can approximate or reconstruct this BC. First, the pseudo-vacuum $\mathrm{BC}$, expressed as

$$
\frac{\partial B_{\text {normal }}}{\partial n}=0, \quad B_{\text {tangential }}=0
$$

can be applied at the interface between the flowcontaining wall and the non-conducting exterior (vacuum). Second, a decay function of the form $\mathbf{B} \propto r^{-n}$ can be used where $r$ and $n$ are the distance from the flow domain and a decay parameter. Third, the Boundary Element Method solves for the magnetic field using a magnetic scalar potential, obtained from a Laplace equation by applying the magnetic field curl-free property outside the flow domain and the divergence-free constraint [5].

In this study we use the pseudo-vacuum BCs as this approach was successfully utilized in commercial solvers ANSYS and FLUENT and can produce physically realistic results for a wide range of $\mathrm{MHD}$ problems [6-8].

\section{Numerical Procedure}

Our code, MOONS, solves the governing equations (1-4) in rectilinear coordinates. Second order accurate finite-difference schemes are used to approximate all spatial derivatives on a staggered grid (Fig. 1). Centered difference stencils were used to compute derivatives, including the advection term in Eq. (1). Non-uniform grids are generated using Robert's stretching functions defined in [9], which ensure that a sufficient number of cells are present in boundary layers.

Momentum and induction equations are solved separately at each time level, where first order accurate explicit time marching is used for all terms except pressure. Pressure is treated purely implicitly. A projection method is used to enforce a divergencefree velocity field, where Gauss-Seidel method is used to iteratively solve the pressure Poisson equation [10]. Diagonal Preconditioned Conjugate Gradient method was used in place of Gauss-Seidel for Shercliff and Hunt flows.

The CT method, described in Ref. [3], is implemented and enforces Eq. (4) within machine accu- 


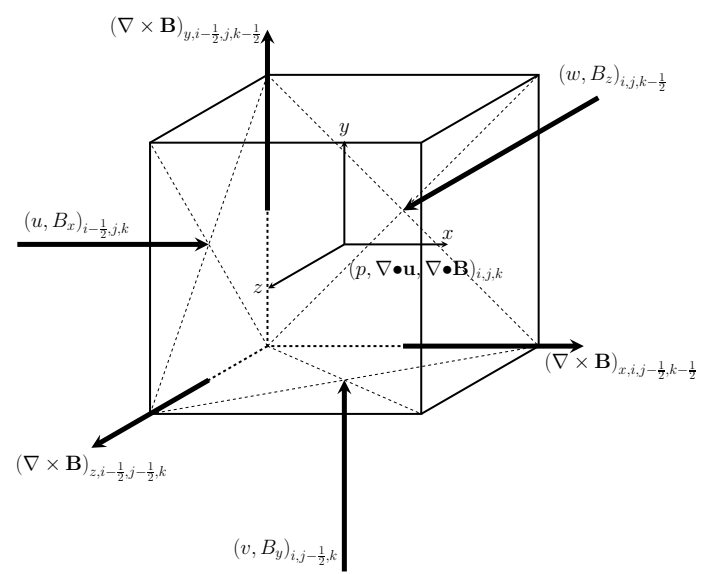

Figure 1: Staggered variables on computational cell.

racy at every time step as long BCs are compatible with Eq. (4) and initial conditions satisfy Eq. (4). The main idea is that $\mathbf{B}$ and $\nabla \times \mathbf{B}$ are staggered on cell faces and edges respectively (Fig. 1), resulting in perfect numerical cancellation when computing Eq. (4). Although not shown in Fig. 1, $\mathbf{u} \times \mathbf{B}$ is also located on cell edges. Interpolations of variables between different cell locations are performed with second order accuracy.

\section{Verification Test Cases}

Several verification tests were conducted including: 1) purely hydrodynamic flows, 2) MHD flows at low $R e_{m}$ and 3) MHD flows at finite $R e_{m}$. The goal of the purely hydrodynamic verification tests was to address grid refinement, spatial order of accuracy and benchmark the hydrodynamic component of MOONS against available numerical solutions.

\subsection{Hydrodynamic Lid-Driven Cavity Flow}

A lid-driven cavity flow is a classic benchmark for fluid dynamic codes due to the complex flow features including transition to turbulence at high Reynolds numbers, development of vortex structures and singularities between the moving lid and the top walls.

This test was to address spatial order of accuracy as suggested in $[11,12]$. A 3D lid-driven cavity flow was simulated for grid resolutions $N_{\text {cells }}=$ $\left(32^{3}, 64^{3}, 128^{3}\right)$ in a domain size $(0 \leq x, y, z \leq 1)$ for $R e=400$. Neumann BCs were used for pressure and all walls were stationary except the driven lid at $y=1$ (Fig. 2). Solutions were compared on all coinciding cell corners of the coarsest grid. The spatial order of accuracy, $p$, and a parameter, $\alpha$ (estimates how far the solution is from the asymptotic range [11]), were computed from

$$
p=\frac{\ln \frac{\left|f^{h_{3}}-f^{h_{2}}\right|}{\left|f^{h_{2}}-f^{h_{1}}\right|}}{\ln (2)}, \quad \alpha=\frac{\left|f^{h_{1}}\right|}{\left|f^{h_{2}}\right|} \frac{\left|f^{h_{3}}-f^{h_{2}}\right|}{\left|f^{h_{2}}-f^{h_{1}}\right|} .
$$

Here, $f^{h_{1}}, f^{h_{2}}, f^{h_{3}}$ are solutions for finest, moderate and coarsest grids, $h_{1}, h_{2}, h_{3}$. The solution is in the asymptotic range when $\alpha / 2^{p}$ is near unity. Spatial order of accuracy and $\alpha / 2^{p}$ for $x, y$ and $z$ components of velocity were $1.72,1.63,1.85$ and 1.0026 , 1.021, 1.014 respectively using $L_{2}$ norms. Taking

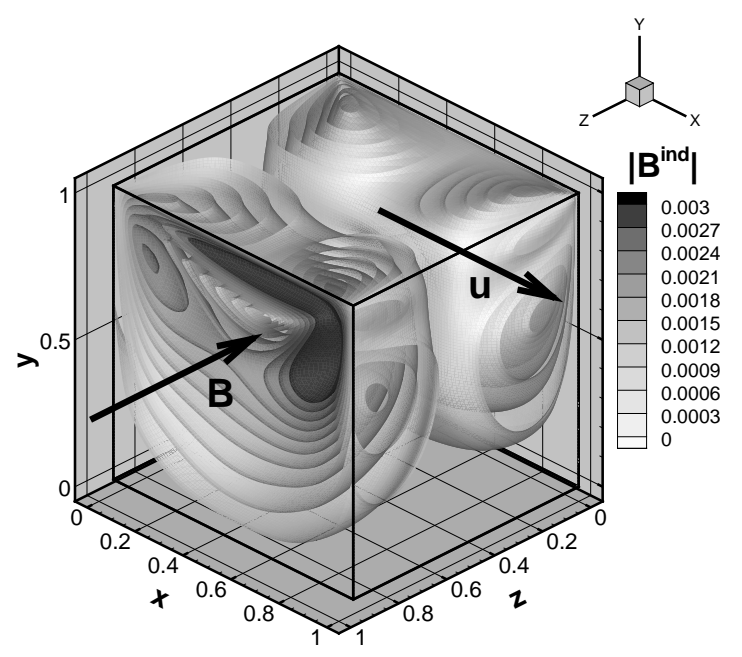

Figure 2: Iso-surfaces of $\left|\mathbf{B}^{\text {ind }}\right|$ between $(0 \leq z \leq 0.94)$ for lid-driven cavity flow.

into considering singularities in the flow, computed values of $p$ and $\alpha / 2^{p}$ are a good indication that the expected order of accuracy is being achieved and that results are in the asymptotic range respectively.

After confirming that the expected order of accuracy was achieved, further computations were performed for $R e=400$ and $R e=1000$ and compared with numerical data in [13]. From rest, both solutions reach a steady state and Fig. 3 shows an excellent match between numerical data for both $R e$. This demonstrates the versatility of MOONS because these two flows have different features. The large centralized vortex, and a second small vortex near $x=1, y=0$, have different shapes for each $R e$ and a third vortex develops in the $x, y=0$ corner for $R e=1000$. 


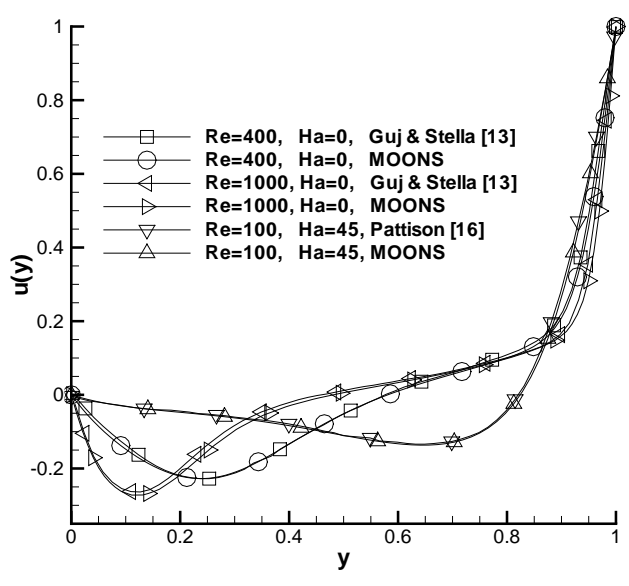

Figure 3: u-component velocity comparison in cavity center.

\subsection{Low $\operatorname{Re}_{m}$ MHD Flows}

Hunt [14], Shercliff [15] and lid-driven cavity MHD flows are presented in this section. A 3D lid-driven cavity MHD flow was simulated and compared with numerical data in [16] for $R e=100$ and $H a=45$ in a domain size $(0 \leq x, y, z \leq 1)$ with $N_{\text {cells }}=64^{3}$.

The applied magnetic field, directed along the $z$ axis (Fig. 2), suppresses motion along its axis and, as a result, the flow tends to a $2 \mathrm{D}$ configuration in the $x y$-plane except in the Hartmann layers near $z=0,1$. The damping effect associated with the Hartmann layers results in more localized motion near the moving lid (Fig. 3) and accelerates temporal flow development. Again, an excellent match is seen between numerical data and present computations in Fig. 3. Interestingly, high velocity and high magnetic field zones roughly coincide (Fig. 2).

Hunt and Shercliff flows were simulated for $R e=$ 10 and $H a=500$. These tests, among others, were suggested by [1] to validate MHD codes. To simulate these fully developed flows, a finite length duct was considered. In the computations, the duct length was 40 with a cross section of $2 \times 2$ and $N_{\text {cells }}=(45,45,45)$. The inlet and outlet velocity was uniform and fully developed respectively with no-slip walls. Neumann BCs were used for pressure. Hyperbolic tangent functions defined a transverse applied magnetic field with a uniform region of length 30 and fringes at 5 and 35 along the duct. In the case of Hunt's flow, the thickness of the Hartmann walls was 0.01 and the liquid and wall electrical conductivities were equal. Expected

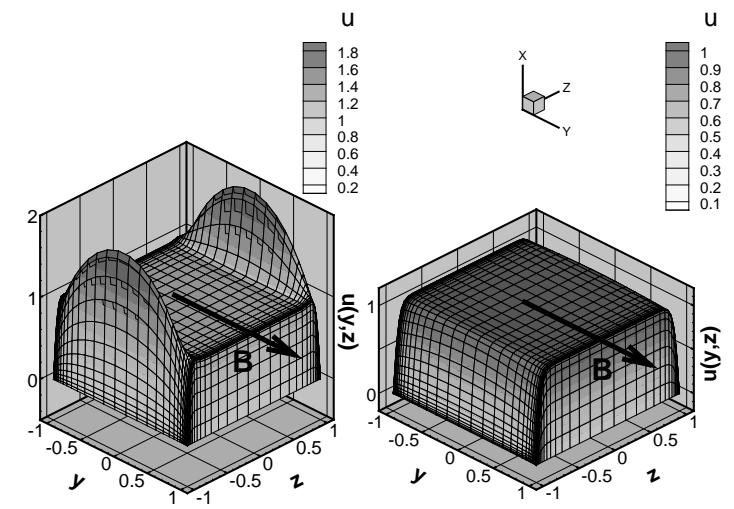

Figure 4: Axial velocity for Hunt (left) and Shercliff (right) flows.

flow characteristics are well captured in both flows. Thin Hartmann boundary layers are observed near the walls perpendicular to the magnetic field with nearly uniform core flow and side wall jets develop in Hunt's flow (Fig. 4). Accurate current and velocity distributions are captured in all flows unlike non-conservative schemes [17].

Error, $\varepsilon$, was computed by comparing dimensionless flow rates, $\tilde{Q}$, with an analytic solution as suggested in [18]:

$$
\varepsilon=\left|\frac{\tilde{Q}_{M O O N S}-\tilde{Q}_{\text {analytic }}}{\tilde{Q}_{\text {analytic }}}\right| \times 100 \% .
$$

The parameter $\tilde{Q}$ was computed in the uniform magnetic field region, far from axial currents generated near the flow inlet and outlet in the fringing field zones. Errors computed from Eq. (7) for Hunt and Shercliff flows were $0.33 \%$ and $1.2 \%$ respectively.

\subsection{Finite $\operatorname{Re}_{m} \mathrm{MHD}$ - Channel Flow}

To verify the finite $R e_{m}$ capabilities of MOONS, a 2D pressure-driven MHD channel flow was simulated and compared with numerical data in [19] for $R e=200, H a=25.82$ and $R e_{m}=1$. The channel was assumed to be infinitely long and periodic in $y$ and $x$ directions respectively with dimensions $(0 \leq x \leq 2 \pi,-1 \leq z \leq 1)$ and $N_{\text {cells }}=(64,64)$. Velocity and induced magnetic field BCs on $z= \pm 1$ were no slip and $\mathrm{B}_{z}=0, \partial \mathrm{B}_{x} / \partial z=0$, enforcing zero current.

The applied magnetic field resembles one of opposing magnets with opposite sign (Fig. 5a). The 

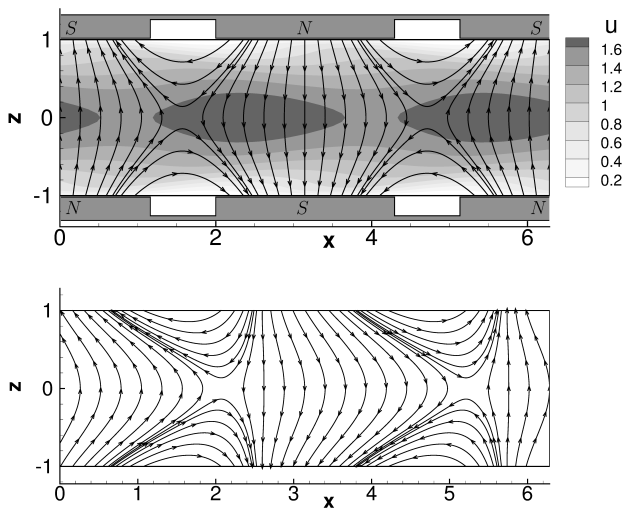

(b)

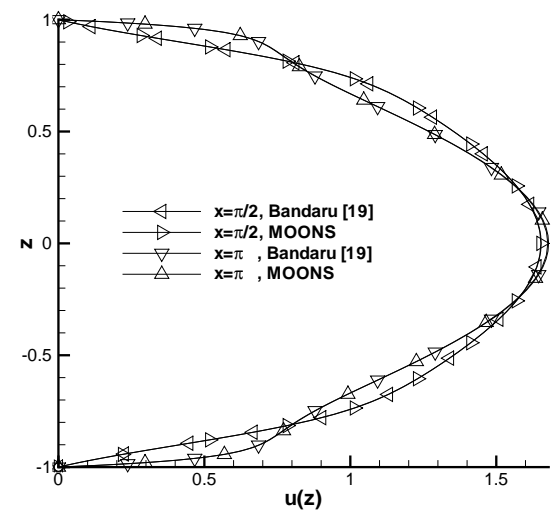

(c)

Figure 5: (a) axial velocity and lines of $\mathbf{B}^{0}$. (b) lines of $\mathbf{B}^{0}+\mathbf{B}^{\text {ind }}$. (c) axial velocity comparison at $x=\pi / 2, \pi$.

fluid drags the magnetic field lines (Fig. 5b), resulting in a distorted overall magnetic field. Similarly, the magnetic field retards fluid motion with spatial periodicity, resulting in periodic fluid motion (Fig. 5a). An excellent match between numerical data is seen in Fig. 5c, where axial velocity distributions are shown at different locations along the channel.

\section{Conclusion Remarks}

A 3D finite-difference B-formulation code was developed and tested for hydrodynamic and MHD flows. Verification showed good results against other numerical codes and analytic solutions for both low and finite $R e_{m}$ flows.

The B-formulation and CT method provide a robust and convenient approach to solve more general MHD problems than the $\varphi$-formulation and naturally enforce important conservation properties respectively. Among the simulated flows, finite $R e_{m}<1$ flows were most time consuming because fast magnetic diffusion and explicit time marching restricted the time step size. In the future, implicit methods may be investigated to solve the induction equation and MOONS will be primarily used to study MHD flows in the presence of truly unsteady magnetic fields.

\section{Acknowledgments}

The first author would like to thank V. Bandaru for numerical data to compare against, shown in Fig. 5c. This material is based upon work supported by the U.S. Department of Energy, Office of Science, Office of Fusion Energy Sciences, under Award Number DE-FG02-86ER52123.

\section{References}

[1] S. Smolentsev, S. Badia, R. Bhattacharyay, et al., Fusion Eng. Des. (2014).

[2] M.-J. Ni, J.-F. Li, J. Comput. Phys. 231 (2012) 281-298.

[3] G. Tóth, J. Comput. Phys. 161 (2000) 605-652.

[4] J. Brackbill, J. Comput. Phys. 430 (1980) 426-430.

[5] A. Iskakov, S. Descombes, E. Dormy, J. Comput. Phys. 197 (2004) 540-554.

[6] G. Rudiger, Y. Zhang, Astron. Astrophys. 378 (2001) 302-308.

[7] S. Kenjeres, K. Hanjalic, Phys. Rev. Lett. 98 (2007) 4-7.

[8] A. Brandenburg, A. Nordlund, R. F. Stein, U. Torkelsson, Astrophys. J. 446 (1995) 741.

[9] R. H. Pletcher, J. C. Tannehill, D. Anderson, Computational Fluid Mechanics And Heat Transfer, CRC Press, 2012.

[10] A. J. Chorin, Math. Comp. 22 (1968) 745.

[11] P. J. Roache, Annu. Rev. Fluid Mech. 29 (1997) 123-160.

[12] P. J. Roache, 41st Aerosp. Sci. Meet. Exhib. 408 (2003) $1-21$.

[13] G. Guj, F. Stella, J. Comput. Phys. 298 (1993) 286-298.

[14] J. C. R. Hunt, J. Fluid Mech. 21 (1965) 577-590.

[15] J. A. Shercliff, Proc. Camb. Phil. Soc. 49 (1953) 136.

[16] M. J. Pattison, K. N. Premnath, N. B. Morley, M. Abdou, Fusion Eng. Des. 83 (2008) 557-572.

[17] H. Araseki, J. Comput. Phys. 110 (1994) 301-309.

[18] S. Smolentsev, N. Morley, M. Abdou, Fusion Eng. Des. 73 (2005) 83-93.

[19] V. Bandaru, J. Pracht, T. Boeck, J. Schumacher, Theor. Comp. Fluid Dyn. (2015). 